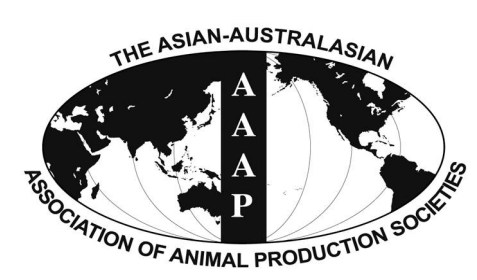

\title{
Rapamycin Inhibits Expression of Elongation of Very-long-chain Fatty Acids 1 and Synthesis of Docosahexaenoic Acid in Bovine Mammary Epithelial Cells
}

\author{
Zhixin Guo ${ }^{a}$, Yanfeng Wang ${ }^{\text {a }}$, Xue Feng, Chaogetu Bao, Qiburi He, Lili Bao ${ }^{1}$, \\ Huifang Hao*, and Zhigang Wang* \\ College of Life Science, Inner Mongolia University, Hohhot 010021, China
}

\begin{abstract}
Mammalian target of rapamycin complex 1 (mTORC1) is a central regulator of cell growth and metabolism and is sufficient to induce specific metabolic processes, including de novo lipid biosynthesis. Elongation of very-long-chain fatty acids 1 (ELOVL1) is a ubiquitously expressed gene and the product of which was thought to be associated with elongation of carbon (C) chain in fatty acids. In the present study, we examined the effects of rapamycin, a specific inhibitor of mTORC1, on ELOVL1 expression and docosahexaenoic acid (DHA, C22:6 n-3) synthesis in bovine mammary epithelial cells (BMECs). We found that rapamycin decreased the relative abundance of ELOVL1 mRNA, ELOVL1 expression and the level of DHA in a time-dependent manner. These data indicate that ELOVL1 expression and DHA synthesis are regulated by mTORC1 in BMECs. (Key Words: Bovine Mammary Epithelial Cells [BMECs], Mammalian Target of Rapamycin Complex 1 [mTORC1], Elongation of Very-long-chain Fatty Acids 1 [ELOVL1], DHA)
\end{abstract}

\section{INTRODUCTION}

Mammary gland biology is often studied in vitro and primary bovine mammary epithelial cells (BMECs) are often used to study protein synthesis and lipid metabolism in the udder of dairy cows. Milk protein, fat content and milk fatty acids (FA) composition are the important indicators for milk quality measurement. Cow's milk fat is the most variable component of milk and primarily composed of triacylglycerol (McFadden and Corl, 2009). Bovine milk contains about $3.5 \%$ to $5 \%$ total lipids, which about $95 \%$ to $98 \%$ or more of the lipid is triacylglycerol and the rest is made up of cholesterol, cholesteryl ester, 1,2diacylglycerol, free fatty acids, monoacylglycerol and phospholipid (Jensen et al., 1991; German and Dillard,

\footnotetext{
* Corresponding Author: Huifang Hao. E-mail: haohf@life.imu.edu.cn / Zhigang Wang. Tel: +86-471-4995867 (8008), Fax: +86-471-4992435, E-mail:1swzg@imu.edu.cn

${ }^{1}$ College of Basic Medical Science, Inner Mongolia Medical University, Hohhot 010110, China.

${ }^{\mathrm{a}}$ These authors contributed equally to this work.

Submitted Aug. 8, 2015; Revised Nov. 11, 2015; Accepted Jan. 5, 2016
}

2006).

FAs are components of most cellular lipids, such as glycerolipids, sphingolipids and cholesterol esters. FAs are classified according to their carbon (C) chain-length, including long-chain fatty acids (LCFAs) with carbon (C) chain lengths of 11/12-20, of which C16 and C18 LCFAs are the most abundant FA species in mammalian cells. The definition of very long-chain fatty acids (VLCFAs) include FAs with $C \geq 22, C \geq 24$, and $C \geq 26$, and are less abundant than LCFAs (Kihara, 2012; Sassa and Kihara, 2014). VLCFAs with C22 and C24 are ubiquitous throughout the body, and VLCFAs with $\mathrm{C} \geq 26$ are often sub-classified into ultra longchain fatty acids (ULCFAs) which found in specific tissues, including the skin, retina, meibomian gland, testis, and brain (Sassa and Kihara, 2014). VLCFAs are classified into saturated fatty acids (SFAs), monounsaturated fatty acids (MUFAs) and polyunsaturated fatty acids (PUFAs), each of which exhibit distinct functions and properties (Ohno et al., 2010; Benoit et al., 2015).

The formation of VLCFA is effected primarily by endoplasmic reticulum (ER) membrane-embedded enzymes, 
including elongases (elongation of very long chain fatty acids, ELOVL), 3-ketoacyl-CoA reductase (KAR), 3hydroxyacyl-CoA dehydratase and trans-2, 3-enoyl-CoA reductase (TER). FA elongation occurs by cycling through a 4-step process (condensation, reduction, dehydration, and reduction), corresponding to the four individual enzymes. In the ELOVL, the first step is rate-limiting (Jakobsson et al., 2006; Kihara, 2012) and elongase is the rate-limiting enzyme during the sequential VLCFA elongation cycle. Seven elongases (ELOVL1-7) have been identified and characterized in mammals, each of which has a specific substrate and function (Kihara, 2012; Xue et al., 2014).

ELOVL1 is a ubiquitously expressed gene, the product of which was thought to be associated with synthesis of C24 sphingolipids (Tvrdik et al., 2000; Ohno et al., 2010). Sphingolipids abound in the plasma membrane and are important in maintaining membrane functions, and ELOVL1 is the chief FA elongase that mediates the production of $\mathrm{C} 24$ sphingolipids in mammalian cells (Ohno et al., 2010; Kihara, 2012). The regulation of ELOVL1 have not been well understood although it is highly expressed in tissues (Jakobsson et al., 2006). It was reported that ELOVL1 activity depends on ceramide synthase 2 (CerS2) expressions (Ohno et al., 2010), and vice versa (Hartmann et al., 2013), which suggested it may form complexes with other related proteins to play an elongase function. A recent study demonstrated that oleic and erucic acids inhibit ELOVL1 and decrease the levels of sphingomyelin with a saturated very-long-chain fatty acid (Sassa et al., 2014) in humans. In contrast, ELOVL1, peroxisome proliferatoractivated receptor (PPAR), and sterol regulatory element binding protein (SREBP) are upregulated in highfat-diet-fed mice (Kozawa et al., 2011). However, the regulatory mechanisms of ELOVL1 expression remain unknown.

Mammalian target of rapamycin (mTOR) is a central regulator of cell growth and metabolism and sufficient to induce specific metabolic processes, including de novo lipid biosynthesis (Düvel et al., 2010; Lodhi et al., 2011; Lamming and Sabatini, 2013). A gene set enrichment analysis of a large set of genes by microarray showed that mammalian target of rapamycin complex 1 (mTORC1) induces ELOVL1 expression in Tsc2 ${ }^{-/-}$cells (Düvel et al., 2010), suggesting that ELOVL1 is a downstream regulatory target of mTORC1. It is believed that docosahexaenoic acid (DHA, C22:6 n-3) is beneficial to human health and the reproductive processes of dairy cows. The concentrations of DHA is inferior to other fatty acids in milk fat of lactating cows (Nogalski et al., 2012). The role of mTORC1 in DHA synthesis is unknown.

To study the role of mTOR signaling pathway in BMECs, we examined the effects of rapamycin, a specific inhibitor of mTORC1, on ELOVL1 expression and DHA synthesis.

\section{MATERIALS AND METHODS}

\section{Primary BMECs culture and treatment}

Primary BMECs were cultured and purified according to previously reported methods (Wang et al., 2013). Purified BMECs were cultured in DMEM/F12 media (DMEM/F12, Gibco, Paisley, PA49RF, Scotland, UK) containing 10\% fetal bovine serum (Hyclone Laboratories, Inc. Logan, UT, USA), bovine insulin ( $5 \mu \mathrm{g} / \mathrm{mL}$, Sigma-Aldrich, Inc. St. Louis, MO, USA), hydrocortisone (1 $\mu \mathrm{g} / \mathrm{mL}$, SigmaAldrich, Inc., USA), progesterone $(1 \mu \mathrm{g} / \mathrm{mL}$, Sigma-Aldrich, Inc., USA), transferrin (5 $\mu \mathrm{g} / \mathrm{mL}$, Sigma-Aldrich, Inc., USA), glutamine (200 mM, Sigma-Aldrich, Inc., USA), EGF (10 ng/mL, Sigma-Aldrich, Inc., USA), $100 \mathrm{U} / \mathrm{mL}$ penicillin $\mathrm{G}$, and $100 \mathrm{mg} / \mathrm{mL}$ streptomycin (Sigma-Aldrich, Inc., USA). Purified BMECs were maintained and incubated in a $25 \mathrm{~cm}^{2}$-tissue culture flask at $37^{\circ} \mathrm{C}$ in humidified air with $5 \% \mathrm{CO}_{2}$. Morphology was examined by light microscopy. For experimental assays, BMECs in the logarithmic growth phase were used in drug treatment.

\section{MTT assay}

Exponentially growing BMECs were used to seed 96well plates at $4 \times 10^{3}$ cells per well $48 \mathrm{~h}$ before drug treatment. Then, cells were incubated with rapamycin at 50 $\mathrm{nM}$ and $100 \mathrm{nM}$ concentrations for $0 \mathrm{~h}, 8 \mathrm{~h}, 24 \mathrm{~h}, 48 \mathrm{~h}$, and $72 \mathrm{~h}$, respectively. The medium with rapamycin was absorbed, and fresh medium was added. 3-(4,5dimethylthiazol-2-yl)-2,5-diphenyltetrazolium bromide (MTT, $5 \mathrm{~g} / \mathrm{L}$; Sigma-Aldrich, USA) was added to each well and incubated for $4 \mathrm{~h}$ at $37^{\circ} \mathrm{C}$. The solution was absorbed, and formazan product was dissolved by adding $100 \mu \mathrm{L}$ DMSO to each well and incubated it for $10 \mathrm{~min}$ at $37^{\circ} \mathrm{C}$. MTT absorbance was measured at 490/630 nm with a spectrophotometer set (Thermo Fisher Scientific, Multiskan SX 353, Waltham, MA, USA).

\section{Reagents and antibodies}

Rapmycin, a specific inhibitor of mTOR, was purchased from Gene Operation (Ann Arbor, MI, USA), and dissolved in ethanol (Sigma-Aldrich, Inc., USA) to a stock concentration of $10 \mu \mathrm{M}$, stored at $-20^{\circ} \mathrm{C}$, and further diluted to an appropriate final concentration with culture medium before use. Ethanol in the final solution did not exceed $0.1 \%(\mathrm{v} / \mathrm{v})$. Antibody to $\beta$-actin was purchased from Sigma Chemical (Sigma-Aldrich, Inc., USA) and Antibody to ELOVL1 was purchased from Abcam plc. (Cambridge, UK). 


\section{Real-time quantitative polymerase chain reaction}

Real-time quantitative polymerase chain reaction (qPCR) was performed to determine the abundance of ELOVL1 mRNA in BMECs of treated groups and control. ELOVL1 was amplified with the following primers: forward 5'- GCT CAG CCC TAC CTT TG -3' and reverse 5'- CCT GGA ATC CCG TTT TG -3'. $\beta$-actin was used as an internal control to normalize RNA loading, and the primers were designed, based on the Bos taurus $\beta$-actin sequence (GenBank Accession number NM_173979.3). $\beta$-actin was amplified with the following primers: forward 5'AGGCACCAGGGCGT-3' and reverse 5'-CCTCGGTCAG CAGC-3'.

Quantitative real-time PCR was performed on a Rotor Gene Q (Qiagen, Hilden, Germany) using SYBR Premix Ex Taq (Perfect Real Time). One microliter of cDNA was amplified in a $25-\mu \mathrm{L}$ mixture that contained $10 \mathrm{mM}$ forward primer $(0.5 \mathrm{~mL}), 10 \mathrm{mM}$ reverse primer $(0.5 \mu \mathrm{L}), 2 \mathrm{SYBR}$ Premix Ex Taq $(12.5 \mu \mathrm{L})$, and nuclease-free water $(10.5 \mu \mathrm{L})$. The program comprised an initial denaturation step at $95^{\circ} \mathrm{C}$ for $5 \mathrm{~min} ; 40$ cycles at $95^{\circ} \mathrm{C}$ for $5 \mathrm{~s}, 54^{\circ} \mathrm{C}$ for $30 \mathrm{~s}$, and $72^{\circ} \mathrm{C}$ for $20 \mathrm{~s} ; 72^{\circ} \mathrm{C}$ for $10 \mathrm{~min}$; and a final melting step. Three technical replicates were run. Delta CT $(\Delta \mathrm{CT})$ values were calculated to determine tissue-specific expression. The real-time PCR results were analyzed by one-way analysis of variance to compare expression between groups.

\section{Enzyme-linked immunosorbent assay}

Purified BMECs were seeded into 24-well plates at $2.35 \times 10^{5}$ cells/well and incubated for growth to $80 \%$ confluence, and then treated with $100 \mathrm{nM}$ rapamycin for 8 $\mathrm{h}$ and $24 \mathrm{~h}$. The medium was absorbed for DHA detection and cells were collected and lysed in PBS by repeated freeze-thaw cycles, centrifuged to remove cellular debris, and assayed immediately or stored at $-80^{\circ} \mathrm{C}$ until analysis for ELOVL1 detection. DHA was measured using the enzyme-linked immunosorbent assay (ELISA) kit (Qian Bi Biology Technology CO., LTD, Shanghai, China) and ELOVL1 was measured using the ELISA kit (Cloud-Clone Corp. Houston, TX, USA) according to manufacturer's instructions, and the absorbance was read at $450 \mathrm{~nm}$ and $570 \mathrm{~nm}$ on a Varioskan Flash Multimode Reader (Thermo Fisher Scientific, Pittsburgh, PA, USA).

\section{Western blot analyses}

Purified BMECs were used to seed 6-well plates at $1.25 \times 10^{6}$ cells/well for culture to $80 \%$ conflunce, and then treated with $100 \mathrm{nM}$ rapamycin for $8 \mathrm{~h}$ and $24 \mathrm{~h}$. The cells were lysed in lysis buffer that contained $25 \mathrm{mM}$ Tris- $\mathrm{HCl}$ (pH 7.6), $150 \mathrm{mM} \mathrm{NaCl}, 1 \%$ Nonidet P-40, 1\% sodium deoxycholate, $0.1 \%$ sodium dodecyl sulfate (SDS), protease inhibitor mixture, and phosphatase inhibitors (SigmaAldrich, Inc., USA) and then placed on ice for $10 \mathrm{~min}$. Next, the cell lysates were harvested by scraping and centrifuged at $4^{\circ} \mathrm{C}$ for $10 \mathrm{~min}$ at $13,000 \mathrm{rpm}$.

Equal amounts of cell lysates were separated by SDSpolyacrylamide gel electrophoresis (PAGE) on $10 \%$ polyacrylamide gels and transferred to PVDF membranes, which were then immunoblotted with the designated primary antibodies. The membranes were then treated with horseradish peroxidase-conjugated goat anti-rabbit IgG (immunoglobulin G) or goat anti-mouse IgG (GE Healthcare, Buckinghamshire, UK) and detected using the enhanced chemiluminescence (ECL) detection (Thermo Fisher Scientific, USA) by exposure to X-ray film. The resolved bands were quantified using Gel-Pro Analyzer 4.0 (Media Cybernetics, Inc., Rockville, MD, USA).

\section{Statistical analysis}

Descriptive statistics were generated for all quantitative data, which were expressed as mean \pm standard deviation. Each assay was performed in triplicate.

\section{RESULTS}

\section{Rapamycin inhibits proliferation of BMECs in a dose- and time-dependent manners}

To determine the inhibitory effects of rapamycin on BMECs proliferation, cells were treated with $50 \mathrm{nM}$ and $100 \mathrm{nM}$ rapamycin for $8 \mathrm{~h}, 24 \mathrm{~h}, 48 \mathrm{~h}$, and $72 \mathrm{~h}$, respectively. The relative cell viability of BMECs was examined by MTT assay. The results showed that rapamycin had inhibitive effect on cell proliferation in a dose- and time- dependent manners (Figure 1), indicating mTORC1 plays a key role in BMECs proliferation.

\section{Rapamycin down-regulates the transcription of ELOVL1 in BMECs}

To determine whether mTORC1 regulates the transcription of ELOVL1 in BMECs, we studied the effects of rapamycin on the relative abundance of ELOVL1 mRNA in BMECs. Cells were treated with $100 \mathrm{nM}$ rapamycin for 8 $\mathrm{h}$ and $24 \mathrm{~h}$, and ELOVL1 mRNA was detected by real-time q-PCR. The results showed that rapamycin decreased the relative abundance of ELOVL1 mRNA in the treated BMECs (Figure 2), suggesting that transcription of ELOVL1 was significantly down regulated $(\mathrm{p}<0.01)$.

\section{Rapamycin inhibits ELOVL1 expression in a time- dependent manner}

To determine the inhibition of rapamycin on synthesis of very long-chain fatty acid in BMECs, we measured the expression of ELOVL1 by ELISA and western blot. The results showed that the expression of ELOVL1 was inhibited after treatment with $100 \mathrm{nM}$ rapamycin for $8 \mathrm{~h}$ and $24 \mathrm{~h}$ (Figure 3), indicating that rapamycin inhibits the 


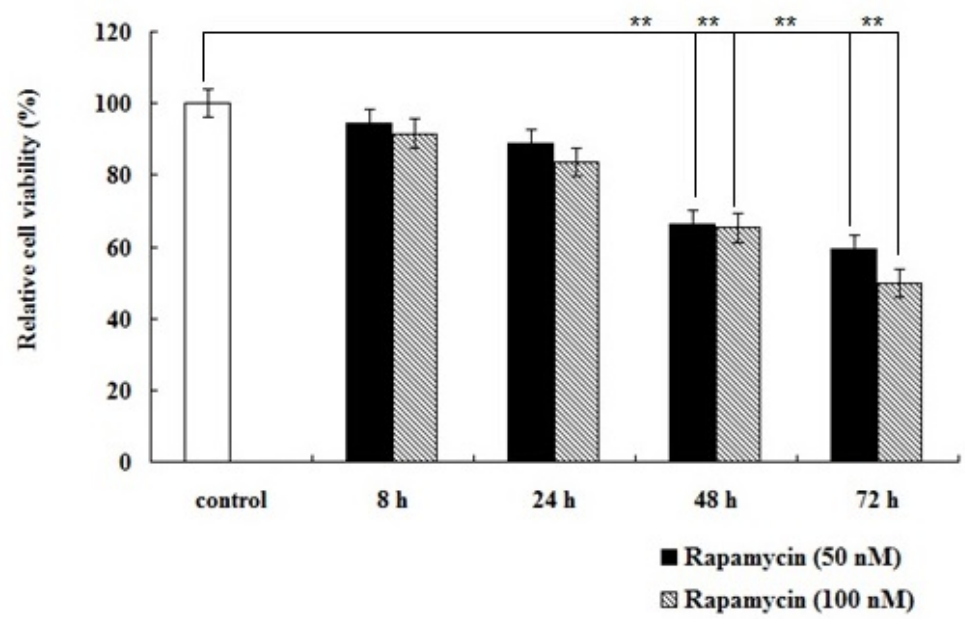

Figure 1. Rapamycin inhibits proliferation of bovine mammary epithelial cells (BMECs). Cells were incubated with rapamycin at $50 \mathrm{nM}$ and $100 \mathrm{nM}$ concentrations for $0 \mathrm{~h}, 8 \mathrm{~h}, 24 \mathrm{~h}, 48 \mathrm{~h}$, and $72 \mathrm{~h}$, respectively. The relative cell viability of BMECs was examined by MTT assay. Rapamycin had inhibitive effect on cell proliferation $(* * \mathrm{p}<0.01)$.

expression of ELOVL1 time-dependently and ELOVL1 expression was regulated by mTORC1.

\section{Rapamycin inhibits DHA synthesis in BMECs}

To determine whether mTORC1 regulates the DHA synthesis in BMECs, we examined the effects of rapamycin on DHA secretion at different times. Cells were treated with $100 \mathrm{nM}$ rapamycin for $8 \mathrm{~h}$ and $24 \mathrm{~h}$, and DHA in medium was detected by ELISA. The results showed that rapamycin produced a time-dependent decrease in the secretion of DHA (Figure 4), suggesting that mTORC1 has function in DHA synthesis in BMECs.

\section{DISSCUSSION}

The first step of VLCFA synthesis is condensation, which is carried out by one of ELOVL1-7, each with different FA substrate preferences. Human ELOVL1 is the functional orthologue of yeast ELO3 and this is in line with that of mouse ELOVL1 (Tvrdik et al., 2000; Ofman and Dijkstra, 2010). ELO3 is recognized as an enzyme which catalyzes biosynthesis of VLCFAs and sphingolipids in yeast, and mammalian orthologues may be equally important in membrane-related functions with regard to sphingolipids (Jakobsson et al., 2006). There exist limited data on how ELOVL1 expression is regulated in mammalian cells despite it being expressed ubiquitously. A previous report has demonstrated that mTORC1 promotes lipid biogenesis by regulating the expression of lipogenic genes (Düvel et al., 2010), the products of which are related to SREBPs and PPARs (Laplante and Sabatini, 2012; 2013), indicating that mTOR signaling pathway may play an integral role in lipid metabolism (Soliman, 2011). Some data suggested that the expression of ELOVL genes is associated with SREBPs and PPARs (Wang et al., 2006; Carmona-Antoñanzas et al., 2014; Sassa et al., 2014; Wang

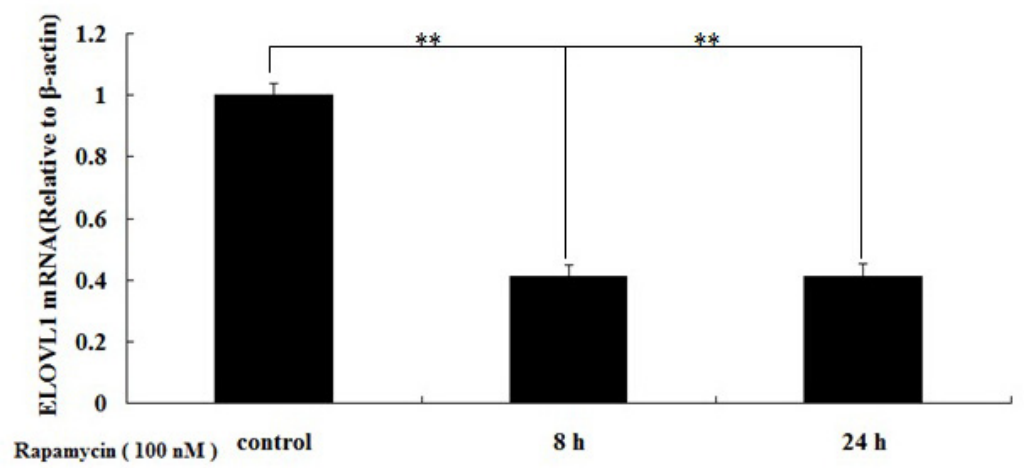

Figure 2. Rapamycin inhibits the relative abundance of elongation of very-long-chain fatty acids 1 (ELOVL1) mRNA in bovine mammary epithelial cells (BMECs). Cells were treated with $100 \mathrm{nM}$ rapamycin for $8 \mathrm{~h}$ and $24 \mathrm{~h}$, and ELOVL1 mRNA was detected by real-time quantitative polymerase chain reaction (qPCR). The relative abundance of ELOVL1 mRNA in the treated BMECs was significantly down regulated $(* * \mathrm{p}<0.01)$. 
(A)

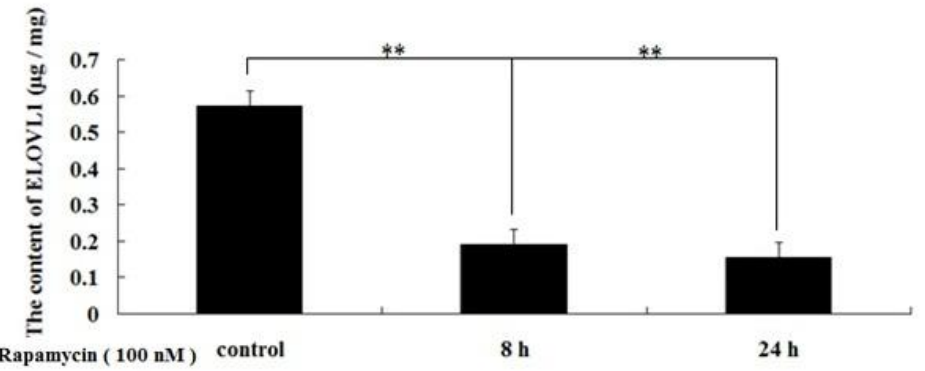

(B)

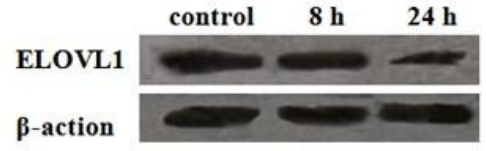

Rapamycin ( $100 \mathrm{nM})$

(C)

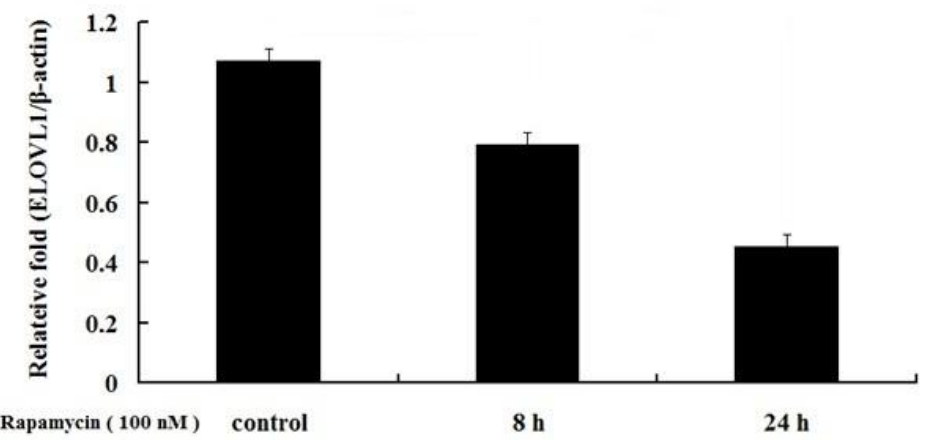

Figure 3. Rapamycin inhibits elongation of very-long-chain fatty acids 1 (ELOVL1) expression in a time-dependent manner. Cells were treated with $100 \mathrm{nM}$ rapamycin for $8 \mathrm{~h}$ and $24 \mathrm{~h}$, and ELOVL1 expression was detected by enzyme-linked immunosorbent assay (ELISA) (A) and Western blot (B). (C) The resolved bands of western blot were quantified using Gel-Pro Analyzer 4.0 (Media Cybernetics, Inc., Rockville, MD, USA). ELOVL1 was down regulated with increasing treatment times $(* * \mathrm{p}<0.01)$.

et al., 2014a) and the expression of ELOVL1 and ELOVL5 was induced by mTORC1, and that mTORC1 activates SREBP1 through S6K1 in MEFs (Düvel et al., 2010). Thus, mTORC1 may regulate the expression of ELOVL genes through the transcription factors SREBP1 and PPAR $\gamma$.
Recent studies in bovines have highlighted a role of mTOR in the regulation of milk synthesis (Zhang et al., 2014), including milk protein synthesis (Wang et al., 2014b; Yu et al., 2014) and triacylglycerol or FAs synthesis (Li et al., 2014). Compared with untreated control cells,

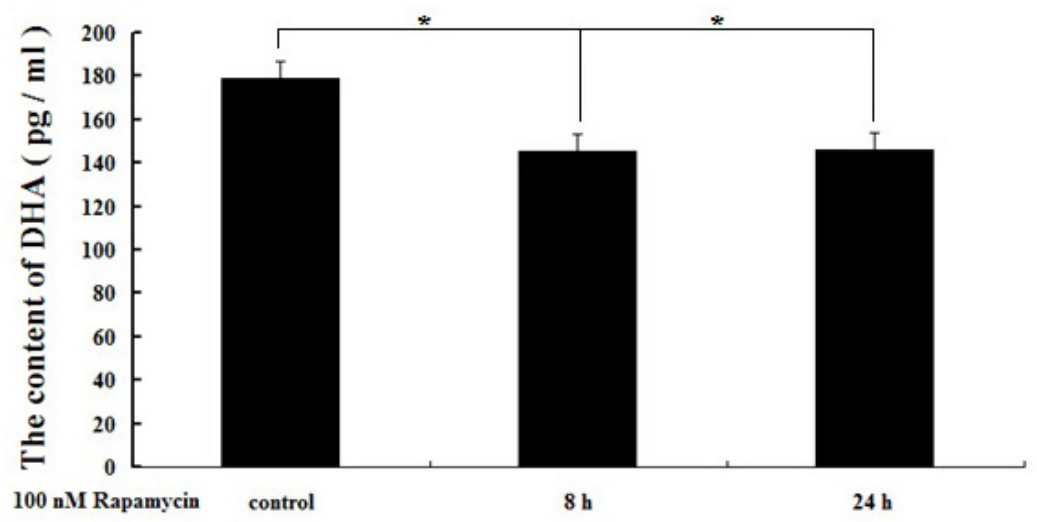

Figure 4. Rapamycin induces a time-dependent decrease in the secretion of DHA in bovine mammary epithelial cells (BMECs). Cells were treated with $100 \mathrm{nM}$ rapamycin for $8 \mathrm{~h}$ and $24 \mathrm{~h}$, and DHA in medium was measured by enzyme-linked immunosorbent assay (ELISA). Rapamycin induced a time-dependent decrease in the secretion of DHA $\left({ }^{*} \mathrm{p}<0.05\right)$. 
rapamycin reduced triglyceride secretion of BMECs (Zhang et al., 2014). Over-expressing of phosphatase and tensin homolog (Pten) gene, a well-established upstream negative regulator of mTOR signaling pathway, inhibits the expression of SREBP1 and PPAR $\gamma$ in BMECs (Wang et al., $2014 b$ ). Therefore mTORC1 may play a key role in the synthesis of milk fat.

Approximately fifty percent of milk FA, including short- and medium-chain FA (C4:0-C14:0) as well as about one-half of the C16:0 are synthesized de novo from acetate and $\beta$-hydroxybutyrate in the mammary gland of dairy cows. The remaining C16:0 and almost all of the longer chain FA are considered to be derived from the diet (Qi et al., 2014) and elongated into long-chain FAs that contain 18 carbon atoms or VLCFAs. In the present study, DHA secretion was down-regulated about $20 \%$ analyzed by ELISA after 8 hours of $100 \mathrm{nM}$ rapamycin treatment (Figure 4), indicating that mTORC1 may play a key role in DHA synthesis. The exact mechanism is not well known and requires to further exploration.

\section{CONCLUSION}

In this study, we examined inhibition of rapamycin on ELOVL1 expression and DHA synthesis in BMECs, and found that rapamycin down-regulated both of them. These data indicate that ELOVL1 expression and docosahexaenoic acid synthesis are regulated by mTORC1 in BMECs.

\section{CONFLICT OF INTEREST}

We certify that there is no conflict of interest with any financial organization regarding the material discussed in the manuscript.

\section{ACKNOWLEDGMENTS}

This study was supported by grants from the Natural Sciences Foundation of China (NO. 31360561), the Natural Science Foundation of Inner Mongolia Autonomous Region of china (2016MS0303, 2016BS0304) and a research program of innovation team from Inner Mongolia (NMGIRT-A1601).

\section{REFERENCES}

Benoit, B., J. Bruno, F. Kayal, M. Estienne, C. Debard, R. Ducroc, and P. Plaisancié. 2015. Saturated and unsaturated fatty acids differently modulate colonic goblet cells in vitro and in rat pups. J. Nutr. 145:1754-1762.

Carmona-Antoñanzas, G., D. R. Tocher, L. Martinez-Rubio, and M. J. Leaver. 2014. Conservation of lipid metabolic gene transcriptional regulatory networks in fish and mammals. Gene 534:1-9.
Düvel, K., J. L. Yecies, S. Menon, P. Raman, A. I. Lipovsky, A. L. Souza, E. Triantafellow, Q. Ma, R. Gorski, and S. Cleaver et al. 2010. Activation of a metabolic gene regulatory network downstream of mTOR complex 1. Mol. Cell 39:171-183.

German, J. B. and C. J. Dillard. 2006. Composition, structure and absorption of milk lipids: A source of energy, fatsoluble nutrients, and bioactive molecules. Crit. Rev. Food Sci. Nutr. 46:57-92.

Hartmann, D., M. S. Wegner, R. A. Wanger, N. Ferreirós, Y. Schreiber, J. Lucks, S. Schiffmann, G. Geisslinger, and S. Grösch. 2013. The equilibrium between long and very long chain ceramides is important for the fate of the cell and can be influenced by co-expression of CerS. Int. J. Biochem. Cell Biol. 45:1195-1203.

Jakobsson, A., R. Westerberg, and A. Jacobsson. 2006. Fatty acid elongases in mammals: Their regulation and roles in metabolism. Prog. Lipid Res. 45:237-249.

Jensen, R. G., A. M. Ferris, and C. J. Lammi-Keefe. 1991. The composition of milk fat. J. Dairy Sci. 74:3228-3243.

Kihara, A. 2012. Very long-chain fatty acids: Elongation, physiology and related disorders. J. Biochem. 152:387-395.

Kozawa, S., A. Honda, N. Kajiwara, Y. Takemoto, T. Nagase, H. Nikami, Y. Okano, S. Nakashima, and N. Shimozawa. 2011. Induction of peroxisomal lipid metabolism in mice fed a highfat diet. Mol. Med. Rep. 4:1157-1162.

Laplante, M. and D. M. Sabatini. 2012. mTOR signaling in growth control and disease. Cell 149:274-293.

Lamming, D. W. and D. M. Sabatini. 2013. A central role for mTOR in lipid homeostasis. Cell Metab. 18:465-469.

Laplante, M. and D. M. Sabatini. 2013. Regulation of mTORC1 and its impact on gene expression at a glance. J. Cell Sci. 126:1713-1719.

Li, N., F. Zhao, C. Wei, M. Liang, N. Zhang, C. Wang, Q. Z. Li, and X. J. Gao. 2014. Function of SREBP1 in the milk fat synthesis of dairy cow mammary epithelial cells. Int. J. Mol. Sci. 15:16998-17013.

Lodhi, I. J., X. Wei, and C. F. Semenkovich. 2011. Lipoexpediency: de novo lipogenesis as a metabolic signal transmitter. Trends Endocrinol. Metab. 22:1-8.

McFadden, J. W. and B. A. Corl. 2009. Activation of AMPactivated protein kinase (AMPK) activation of inhibits fatty acid synthesis in bovine mammary epithelial cells. Biochem. Biophys. Res. Commun. 390:388-393.

Nogalski, Z., M. Wroński, M. Sobczuk-Szul, M. Mochol, and P. Pogorzelska. 2012. The effect of body energy reserve mobilization on the fatty acid profile of milk in high-yielding cows. Asian Australas J. Anim. Sci. 25:1712-1720.

Ofman, R. and I. M. E. Dijkstra. 2010. The role of ELOVL1 in very long-chain fatty acid homeostasis and X-linked adrenoleukodystrophy. EMBO Mol. Med. 2:90-97.

Ohno, Y., S. Suto, M. Yamanaka, Y. Mizutani, S. Mitsutake, Y. Igarashi, T. Sassa, and A. Kihara. 2010. ELOVL1 production of C24 acyl-CoAs is linked to C24 sphingolipid synthesis. Proc. Natl. Acad. Sci. USA. 107:18439-18444.

Qi, L., S. Yan, R. Sheng, Y. Zhao, and X. Guo. 2014. Effects of saturated long-chain fatty acid on mRNA expression of genes associated with milk fat and protein biosynthesis in bovine mammary epithelial cells. Asian Australas. J. Anim. Sci. 27:414-421. 
Sassa, T. and A. Kihara. 2014. Metabolism of very long-chain fatty acids: Genes and pathophysiology. Biomol. Ther. (Seoul) 22:83-92.

Sassa, T., T. Wakashima, Y. Ohno, and A. Kihara. 2014. Lorenzo's oil inhibits ELOVL1 and lowers the level of sphingomyelin with a saturated very long-chain fatty acid. J. Lipid Res. 55:524-530.

Soliman, G. A. 2011. The integral role of mTOR in lipid metabolism. Cell Cycle. 10:861-862.

Tvrdik, P., R. Westerberg, S. Silve, A. Asadi, A. Jakobsson, B. Cannon, G. Loison, and A. Jacobsson. 2000. Role of a new mammalian gene family in the biosynthesis of very long chain fatty acids and sphingolipids. J. Cell Biol. 149:707-718.

Wang, Q., M. Tikhonenko, S. N. Bozack, T. A. Lydic, L. Yan, N. L. Panchy, K. M. Mcsorley, M. S. Faber, Y. Yan, M. E. Boulton, M. B. Grant, and J. V. Busik, 2014a. Changes in the daily rhythm of lipid metabolism in the diabetic retina. PLoS One. 9:e95028. doi: 10.1371/journal.pone.0095028. eCollection 2014.

Wang, X., L. Xiu, Q. Hu, X. Cui, B. Liu, L. Tao, T. Wang, J. Wu, Y. Chen, and Y. Chen. 2013. Deep sequencing-based transcriptional analysis of bovine mammary epithelial cells gene expression in response to in vitro infection with staphylococcus aureus stains. PLoS One. 8:e82117.
Wang, Y., D. Botolin, J. Xu, B. Christian, E. Mitchell, B. Jayaprakasam, M. G. Nair, J. M. Peters, J. V. Busik, L. K. Olson, and D. B. Jump. 2006. Regulation of hepatic fatty acid elongase and desaturase expression in diabetes and obesity. J. Lipid Res. 47:2028-2041.

Wang, Z., X. Hou, B. Qu, J. Wang, X. Gao, and Q. Li. 2014b. Pten regulates development and lactation in the mammary glands of dairy cows. PLoS One. 9:e102118. doi: 10.1371/journal.pone.0102118. eCollection 2014.

Xue, X., C. Y. Feng, S. M. Hixson, K. Johnstone, D. M. Anderson, C. C. Parrish, and M. L. Rise. 2014. Characterization of the fatty acyl elongase (ELOVL) gene family, and hepatic elovl and delta- 6 fatty acyl desaturase transcript expression and fatty acid responses to diets containing camelina oil in Atlantic cod (Gadus morhua). Comp. Biochem. Physiol. B. Biochem. Mol. Biol. 175:9-22.

Yu, C., C. Luo, B. Qu, N. Khudhair, X. Gu, Y. Zang, C. Wang, N. Zhang, Q. Li, and X. Gao. 2014. Molecular network including eIF1AX, RPS7, and 14-3-3 $\gamma$ regulates protein translation and cell proliferation in bovine mammary epithelial cells. Arch. Biochem. Biophys. 564:142-155.

Zhang, X., F. Zhao, Y. Si, Y. Huang, C. Yu, C. Luo, N. Zhang, Q. $\mathrm{Li}$, and X. Gao. 2014. GSK3 $\beta$ regulates milk synthesis in and proliferation of dairy cow mammary epithelial cells via the mTOR/S6K1 signaling pathway. Molecules 19:9435-9452. 